\title{
Power harvesting with PZT ceramics and circuits design
}

\author{
Hong Chen · Chen Jia $\cdot$ Wenhan Hao $\cdot$ Chun Zhang • \\ Zhihua Wang $\cdot$ Chunsheng Liu
}

Published online: 26 August 2009

(C) Springer Science+Business Media, LLC 2009

\begin{abstract}
Power generated from a piezoelectric material usually comes with poor characteristics such as high voltage, low current and high impedance. In order to drive the embedded sensor circuit, piezoelectric power needs to be characterized and regulated. In this paper, we present an analysis on the power generation characteristics and the efficiency of power conversion of the stiff lead zirconate titanate (PZT) ceramics. Moreover, a power circuit design is put forward in the application where PZT elements are used for power generation in a TKR implant. A hybrid direct current (DC)-DC, comprising a switched capacitor (SC) DC-DC converter and a low dropout (LDO) linear voltage regulator, is presented. The variable ratio SC converter has been taped out with $0.35 \mu \mathrm{m}$ CMOS technology. The test results show that the SC converter can transfer the input voltage that ranges from 5 to $14 \mathrm{~V}$ from the PZT ceramics into the voltage ranging from 2 to $2.5 \mathrm{~V}$ which will be dealt with by LDO circuit whose efficiency can reach $80 \%$.
\end{abstract}

The online version of the original article can be found under doi: 10.1007/s10470-009-9299-5.

Due to errors in the experimental results section, this article is being reprinted in its entirety. This version contains the corrected experimental results.

H. Chen $(\bowtie) \cdot$ C. Jia $\cdot$ W. Hao $\cdot$ C. Zhang $\cdot$ Z. Wang

Tsinghua National Laboratory for Information Science and

Technology, Institute of Microelectronics, Tsinghua University,

Beijing 100084, China

e-mail: hongchen@tsinghua.edu.cn

C. Liu

Department of Computer and Electronics Engineering,

University of Nebraska-Lincoln, Omaha, NE 68182-0572, USA

e-mail: chunshengliu@unlnotes.unl.edu
Keywords PZT $\cdot$ Power generation $\cdot$ LDO $\cdot$ Power circuit

\section{Introduction and related work}

Battery has been the power source of most electric-driven devices. However, the limited lifetime and physical dimension have rendered traditional batteries unacceptable for some power-critical or maintenance-free real-time embedded applications such as the wireless sensor, orthopedic implants, etc. [1].

Piezoelectric materials can create electrical charge when being mechanically stressed. Various analysis and approaches of energy harvesting using piezoelectric materials have been reported [1-8]. Some works focus on the characteristic of power generation of piezoelectric material $[1,2]$. Other works deal with power optimization and electrical interface circuit of power generation [3-7].

Unregulated energy is used in [1], where a theoretical analysis of power generation for PZT ceramics is presented, which is then verified by simulation and experimental results. Several important considerations in designing such generators are explored, including parameter identification, load matching, form factors, efficiency, longevity, energy conversion, and energy storage. Finally, an application is presented where electrical energy is generated inside a prototype total knee replacement (TKR) implant.

All of the above works except [1] use in-vivo sensors for research purposes only. In contrast, our work aims to use self-powered sensors in real-world clinical implants. Compared to [1], smaller PZT elements are used in our work with a volume of only $0.5 \times 0.5 \times 1.8 \mathrm{~cm}^{3}$ (compared with $1 \times 1 \times 1.8 \mathrm{~cm}^{3}$ in [1]) which specifically addresses the problem of limited space available inside an implant. Hence it presents a more practical application scenario. 
In the application of TKR, implant kinematics require that deflection of the bearing surfaces be small $(<10 \mu \mathrm{m})$ and the usable space inside implants is extremely limited. Therefore, stiff PZT ceramic elements (part number "TS18H5-104" from Piezo Systems [1], http://www.piezo.com/ prodstacks 1.html) are used in this work.

In modern electronic medical systems, the most important trends are low power consumption and integration. First, low power systems make themselves portable, even being implanted in human beings' body. Secondly, the system on a chip of medical instruments can further reduce instruments volume and power consumption. In this paper, we use PZT generator in vivo, in which the PZT elements are not only used to supply power for the wireless monitoring system of the orthopaedic implants (WMSoOI) [9], but also detect the defects of artificial joint through signal variations generated by PZT sensors.

The paper is organized as follows: In Sect. 2, The PZT modeling and the parameter fitting are shown. In Sect. 3, we discuss the efficiency of power conversion of the PZT elements. In Sect. 4, an integrated power generation and regulation circuit using PZT elements is described. The design of the SC converter and LDO linear voltage regulator are also illustrated.

\section{Experiment fit of model parameters}

According to [1] and [8], the equivalent electronic circuit of the PZT is shown in Fig. 1. The detailed analysis can be found in [8] and has been omitted here. In order to determine the parameters in the equivalent electronic circuit of the PZT, a set of experiments were conducted using low frequency (1 Hz) sinusoidal input force waveforms with amplitudes 900 N. A non-linear least square fitting method was performed. The fitting results are: $\phi=0.03, L_{\mathrm{e}}=6 \mathrm{e}-6 \mathrm{H}$, $R_{\mathrm{e}}=0.4$ Ohms, $C_{\mathrm{e}}=0.77 \mathrm{e}-6 \mathrm{~F}$, and $C_{\mathrm{p}}{ }^{\prime}=1.491 \mathrm{e}-6 \mathrm{~F}$. HSPICE is used to simulate the circuit shown in Fig. 1, with the new fitted parameters applied. In [10] the experimental data and the simulation results are given and the two kinds of data match closely. It can be seen that about $0.3 \mathrm{~mW}$ raw power can be generated by one single PZT element.

In order to obtain more power, another set of experiments was performed with four identical PZTs in parallel. The experimental peak power is not as expected, four times as

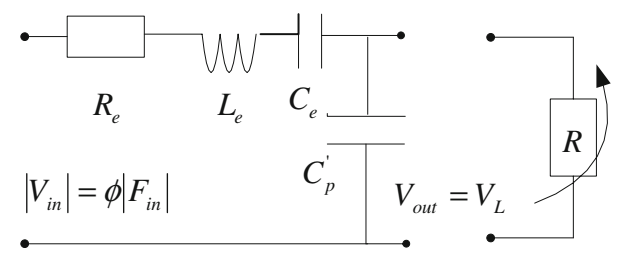

Fig. 1 PZT element modeled as a circuit much as the peak power generated by a single PZT element. This discrepancy can be explained by the fact that the four PZTs receive different amount of force in the experiments, therefore not every PZT can generate the maximum power (about $0.3 \mathrm{~mW}$ ), as a result, the power generated by the four PZTs is $<1.2 \mathrm{~mW}$ (only about $1 \mathrm{~mW}$ ).

\section{Efficiency of power conversion}

From Fig. 1 the efficiency of power conservation can be calculated by the formula (1) as follows:

$\eta=\frac{P_{\text {Load }}}{P_{\text {in }}}$

where $P_{\text {Load }}$ is the average power delivered to the load, and $P_{\text {in }}$ is the input mechanical power. They can be given by formula (2) and (3), respectively

$P_{\text {Load }}=\frac{V_{\text {out }}^{2}}{R}$

$P_{\text {in }}=\frac{V_{\text {in }}^{2}}{Z_{\text {total }}}$

where $V_{\text {out }}$ and $V_{\text {in }}$ are the amplitudes of output voltage and input voltage of the equivalent circuit shown in Fig. 1. $Z_{\text {total }}$ is the total impendence of the equivalent circuit. Under a sinusoidal input force profile, $V_{\text {out }}$ can be given by (4). And $V_{\text {in }}=\phi\left|F_{\text {in }}\right|$.

$V_{\text {out }}=\frac{\left|Z_{\text {part }}\right|}{\left|Z_{\text {total }}\right|} V_{\text {in }}$

$Z_{\mathrm{part}}=\frac{R}{1+j \omega R C_{p}^{\prime}}$

$Z_{\text {total }}=Z_{\text {part }}+R_{\mathrm{e}}+j \omega L_{\mathrm{e}}+\frac{1}{j \omega R C_{\mathrm{e}}}$

where $Z_{\text {part }}$ is the parallel electrical impedance of the piezoelectric and the load $R$.

Using the fitting result discussed in Sect. 2, the relationship between the efficiency and the frequency and the load $R$ can be calculated. The surface of Fig. 2 represents the result. The input force profile was sinusoidal with $900 \mathrm{~N}$ amplitude, and the efficiency was calculated for a range of frequencies and load resistors. The results indicate that the best efficiencies are obtained when the frequency is about $79 \mathrm{kHz}$ and the load is about $89 \mathrm{KOhms}$. As the input frequency increases, the maximum efficiency occurs at smaller load resistance. That's because as the frequency increase the impedance $1 / 1 j \omega C_{\mathrm{p}}{ }^{\prime}$ will decrease.

Although the motion frequency of patients will be far less $79 \mathrm{kHz}$ when the PZT elements are embedded into orthopedic implants, in other words, we will have to make the PZT elements work at a frequency with lower power 


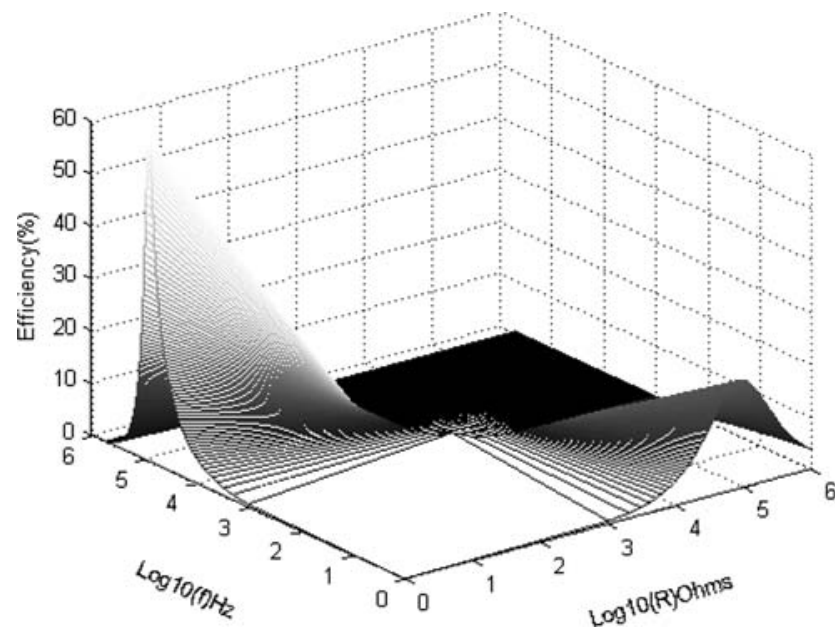

Fig. 2 Efficiency versus frequency and resistive load

conversion efficiency. Nonetheless, we still could maintain a reasonable level of power supply (about $1.2 \mathrm{~mW}$ as said in Sect. 2) by properly choosing load capacitance and resistance values ( $R$ and $C$ ranges from $20 \mathrm{KOhms}$ to $50 \mathrm{KOhms}$ and $>10 \mu \mathrm{F}$, respectively).

\section{Power circuits design}

Considering the output signal of PZT varies with the force in TKR, the power spectrum is calculated using an FFT algorithm. It is found that the piezoelectric signal mainly consists of frequencies between 1 and $4 \mathrm{~Hz}$. At $1 \mathrm{~Hz}$, the power in the signal is a maximum, and at frequencies higher than $4 \mathrm{~Hz}$, the power in the signal approaches zero. The output power of PZT varies with the force applied on the TKR implant. When the input frequency is significantly lower than the resonance frequency of PZT element, the equivalent circuit has a linear transform function in the frequency domain. As a result, the output signal would also follow the input signal and deliver almost no power at frequencies higher than $4 \mathrm{~Hz}$. With such an input signal, the designed power circuit is shown in Fig. 3.

In Fig. 3, the output signal of PZT will be first rectified by a full-wave bridge rectifier to convert the bipolar piezoelectric output to a unipolar signal. Then, it is followed

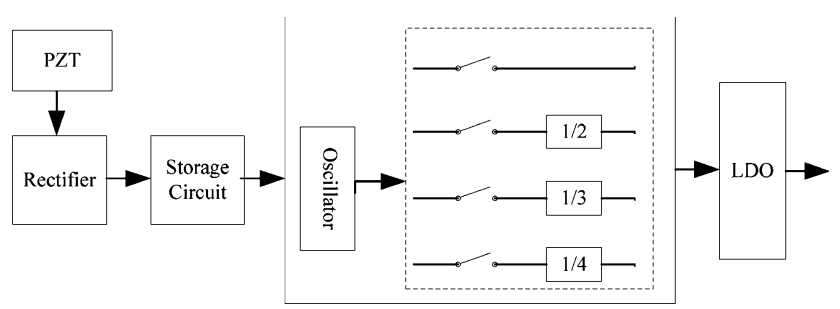

Fig. 3 Schematic power circuit by a "storage circuit" which consists of a capacitor in parallel with the load circuit. An "oscillator" circuit is used to generate clock for an "SC converter" (switching capacitor based on DC-DC converter), which will lower the input voltage from $\sim 10$ to $2 \mathrm{~V}$. The input voltage is controlled by four programmable switches, as shown in the dashed block in Fig. 3. Finally, the designed low drop-out (LDO) voltage regulator drops the voltage further to a steady voltage.

The SC converter circuits have been taped out at the process of $0.35 \mu \mathrm{m}$ CMOS HV technology. The microphotograph of the chip is illustrated in Fig. 4. The test results of the chip are shown in Table 1 . It can be seen that the SC converter can transfer the input voltage that ranges from 5 to $14 \mathrm{~V}$ from the PZT elements into the voltage ranging from 2 to $2.5 \mathrm{~V}$ which will be dealt with by LDO circuit. The total efficiency of the SC converter is from 28 to $42 \%$ at fulltime working mode.

Figure 5 presents the measured power converter efficiency versus input voltage with the maximum load

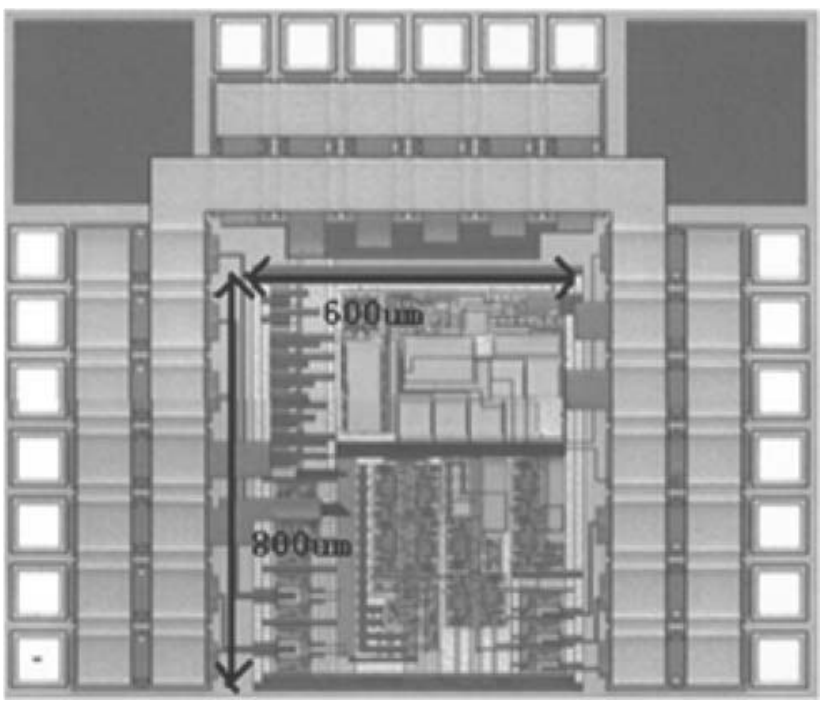

Fig. 4 Die microphotograph of the SC converter

Table 1 Test result of the chip

Input voltage $\left(V_{\text {in }}\right)(5-14 \mathrm{~V})$,

load resistance $(3.85 \mathrm{KOhms})$

Results

Output voltage (V)

$2-2.5$

Quiescent supply current $(\mu \mathrm{A})$

$143-240$

Transmission efficiency (\%)

49-61

Output current $(\mu \mathrm{A})$

$$
\begin{aligned}
& 5.5 \mathrm{~V}<V_{\text {in }}<7.5 \mathrm{~V} \\
& 7.5 \mathrm{~V}<V_{\text {in }}<11.5 \mathrm{~V} \\
& 11.5 \mathrm{~V}<V_{\text {in }}<14 \mathrm{~V}
\end{aligned}
$$$$
560
$$$$
580
$$

Output voltage ripple $\left(\mathrm{mV}_{\mathrm{pp}}\right)$

460

Total power efficiency $(\%)$ 
current. The measured curve shows that the converter efficiency decreases with input voltage before changing the step-down conversion ratio. When the input voltage just exceeds the threshold voltage, the step-down conversion ratio is set to be maximum $2 / 3$. With the input voltage increasing, the converter efficiency goes down, because system energy loss increases with supply voltage. When the input voltage reaches to the settled value, the ratio turns to $1 / 2$ and efficiency bounces up because its no-load output voltage is closer to the load voltage desired. It also happens when the ratio changes from $1 / 2$ to $1 / 3$. The maximum efficiency occurs when the input voltage is $5 \mathrm{~V}$.

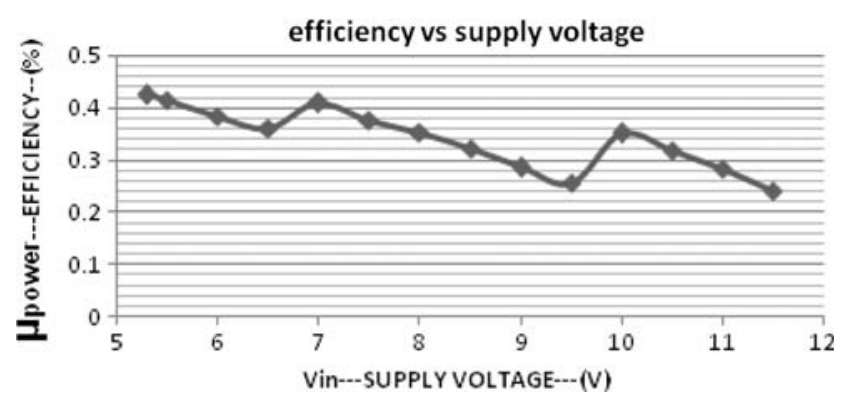

Fig. 5 The measured power converter efficiency varies with different input voltages
Traditional LDO circuit structure consists of three components: an error amplifier, a power transistor and a resistive voltage divider. The positive input of error amplifier is connected to the reference voltage and the negative one is connected to the resistive voltage divider of output voltage. The output voltage of error amplifier is to control the gate voltage of power transistor. Because the subsequent signal circuit includes analogue components, such as $\mathrm{AD}$ converters and RF modules, power-supply rejection ratio (PSRR) of LDO is a very important characteristic, which is a measure of the ability of an output voltage to prevent noise coupled with power supply. On the one hand, high gain of error amplifier will lead to high PSRR; on the other hand, using PMOS transistor as power transistor will reduce power supply noise by offsetting it through power transistor and error amplifier [11].

In this case, error amplifier DC gain is more than $70 \mathrm{~dB}$ and PMOS transistor is used as the power transistor. In order to suppress the supply noise from PMOS transistor, the error amplifier uses NMOS input differential pair and PMOS current-mirror load connected to the supply, as shown in Fig. 6.

Table 2 shows the performances of LDO circuits [12]. Line regulation, load regulation and PSRR are important parameters for LDO design. LDO is expected to output a constant voltage even if input voltage changes a lot. Line

Fig. 6 Concrete LDO circuit

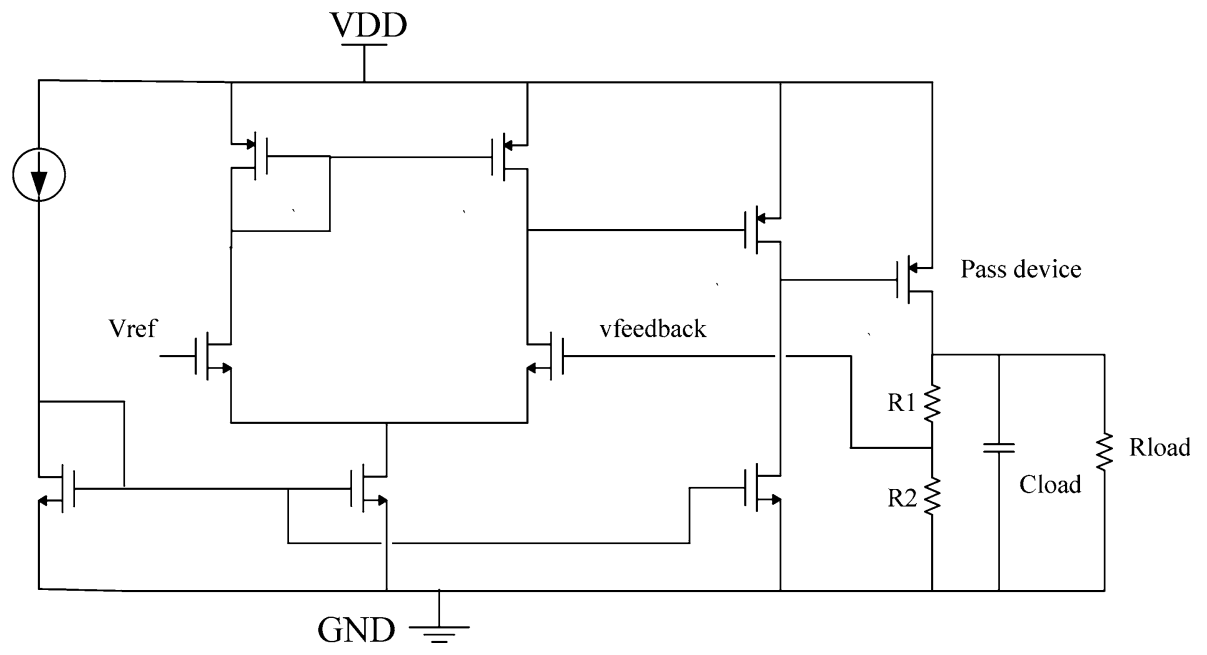

Table 2 Summary of the LDO circuit results

\begin{tabular}{llll}
\hline & Line regulation & Load regulation & PSRR \\
\hline Condition & $2 \mathrm{~V}<$ input voltage $<3 \mathrm{~V}$ & $1 \mathrm{~mA}<$ load current $<5 \mathrm{~mA}$ & Input voltage $=2 \mathrm{~V}$ \\
Typical & $122.99 \mu \mathrm{V}$ & $180.99 \mu \mathrm{V}$ & $41.96 \mathrm{~dB} @ 1 \mathrm{MHz}$ \\
& & & $71 \mathrm{~dB} @ 10 \mathrm{kHz}$ \\
ff & $144.99 \mu \mathrm{V}$ & $170.73 \mu \mathrm{V}$ & $43.05 \mathrm{~dB} @ 1 \mathrm{MHz}$ \\
& & & $73 \mathrm{~dB} @ 10 \mathrm{kHz}$ \\
ss & $207.30 \mu \mathrm{V}$ & $43.06 \mathrm{~dB} @ 1 \mathrm{MHz}$ \\
& $99.16 \mu \mathrm{V}$ & & $72 \mathrm{~dB} @ 10 \mathrm{kHz}$ \\
\hline
\end{tabular}


regulation is such a measure of the ability of the power supply to maintain its output voltage given changes in the input line voltage. Usually, it is simulated by changing input voltage at the range from low power supply to high power supply using DC analysis method. In the same way, LDO is expected to output a constant voltage even if load current changes a lot. Load regulation is a measure of the ability of an output voltage to remain constant given changes in the load. And it is simulated by sweeping load current from small load current to high load current using DC analysis method.

\section{Conclusion}

This paper first presents the model and fitting results of the parameters in the model of the PZT element (part number of TS18-H5-104, Piezo Systems). In order to get more power from such PZT element in the application of TKR, four PZT elements are used. When their positions in the fixer are in a line, maximum power $(\approx 1.2 \mathrm{~mW})$ can be obtained. The efficiency of the power conversion of the PZT is also given. A power circuit including the SC converter and LDO voltage regulator is designed for the monitoring system of the TKR implant, making the system self-powering and suitable for remote operations. Further study on the chip design of the system will be done in the future work.

Acknowledgments The authors are sincerely thankful to the Medical Center of University of Nebraska-Lincoln at Omaha for providing the PZTs used in this study and the lab equipment. This research was supported by the National Natural Science Foundation of China (No. 60475018) and the orthopaedic research lab of the Medical Center of University of Nebraska-Lincoln at Omaha.

\section{References}

1. Platt, S. R., Farritor, S., Garvin, K., \& Haider, H. (2005). The use of piezoelectric ceramics for electric power generation within orthopedic implants. IEEE/ASME Transactions on Mechatronics, 10(4), 455-461.

2. Keawboonchuay, C., \& Engel, T. G. (2003). Electrical power generation characteristics of piezoelectric generator under quasistatic and dynamic stress conditions. IEEE Transactions on Ultrasonics Ferroelectrics and Frequency Control, 50(10), 13771382.

3. Priya, S. (2006). High power universal piezoelectric transformer. IEEE Transactions on Ultrasonics, Ferroelectrics and Frequency Control, 53(1), 23-29.

4. Fung, S. W., \& Pong, M. H. (2004). A microprocessor controlled piezoelectric power converter. The 4th International Power Electronics and Motion Control Conference, 3, 1152-1157.

5. Ottman, G. K., Hofmann, H. F., Bhatt, A. C., \& Lesieutre, G. A. (2002). Adaptive piezoelectric energy harvesting circuit for wireless remote power supply. IEEE Transactions on Power Electronics, 17(5), 669-676.

6. Badel, A., Benayad, A., Lefeuvre, E., et al. (2006). Single crystals and nonlinear process for outstanding vibration-powered electrical generators. IEEE Transactions on Ultrasonics, Ferroelectrics, and Frequency Control, 53(4), 673-684.

7. Le, T. T., Han, J., von Jouanne, A., et al. (2006). Piezoelectric micro-power generation interface circuits. IEEE Journal of SolidState Circuits, 41(6), 1411-1420.

8. Platt, S. R. (2009). Electric power generation within orthopaedic implants using piezoelectric ceramics. Thesis, University of Lincoln.

9. Chen, H., Liu, M., Jia, C., Zhang, C., \& Wang, Z. (2007). Low power IC design of the wireless monitoring system of the orthopedic implants. In Proceeding of 29th annual international conference of the IEEE engineering in medicine and biology society, Lyon, France, pp. 5766-5769.

10. Chen, H., Jia, C., Zhang, C., \& Wang, Z. (2007). Power harvesting with PZT ceramics. In ISCAS 2007. IEEE international symposium on circuits and systems, May 27-30, 2007, New Orleans, LA, pp. 557-560.

11. Gupta, V., \& Rinco'N-Mora, G. A. (2007). A $5 \mathrm{~mA} 0.6 \mathrm{~mm}$ CMOS Miller-compensated LDO regulator with $227 \mathrm{~dB}$ worstcase power-supply rejection using $60 \mathrm{pF}$ of on-chip capacitance (pp. 520-521). San Francisco, CA: ISSCC.

12. Jia, C., Chen, H., Liu, M., Zhang, C., \& Wang, Z. (2008). Integrated power management circuit for piezoelectronic generator in wireless monitoring system of orthopaedic implants. IET Circuit Device and System, 2(6), 485-494.

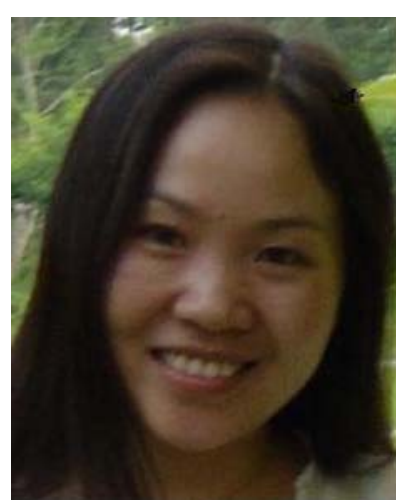

Hong Chen received the B.S. degree and M.S. degree in Communication Engineering Department from Information Engineering University, Zhengzhou, China in 1995 and 1998 respectively. She received the Ph.D. degree in the Department of Electronic Engineering from Tsinghua University in 2005. She is now working in the Institute of Microelectronics in Tsinghua University as an assistant professor. Her research interest include biomedical system design, integrated circuit design, PZT power electronics, SoC, and ASIC design.

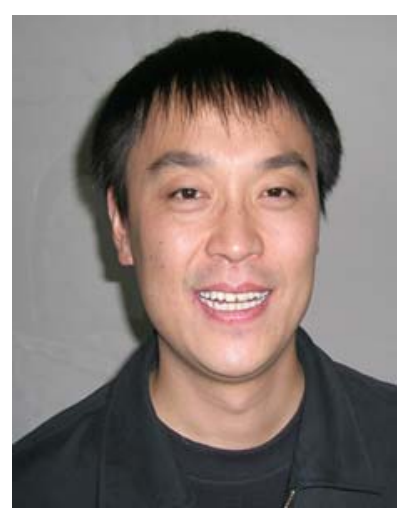

Chen Jia received the B.S. degree in Electrical Engineering from Shanxi University in 1998 and the M.S. degree in Biomedical Engineering from University of Electric Science and Technology of China in 2001. $\mathrm{He}$ is currently working on power management circuits for power harvesting systems. His research interests include power management circuits for portable devices, power harvesting systems and display drivers. 

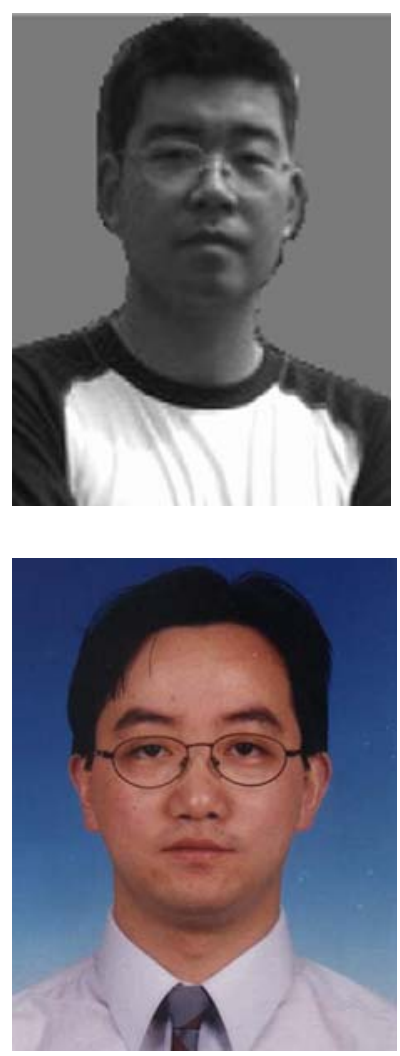

Chun Zhang (S'00-M'03) received the B.S., M.S., and $\mathrm{Ph} . \mathrm{D}$. degrees from the Department of Electronic Engineering, Tsinghua University, Beijing, China, in 1995, 1997, and 2000, respectively. He became an Associate Professor of the Institute of Microelectronics of Tsinghua University in 2005. His major research fields are signal processing, design of mixed signal integrated circuit and RFID.

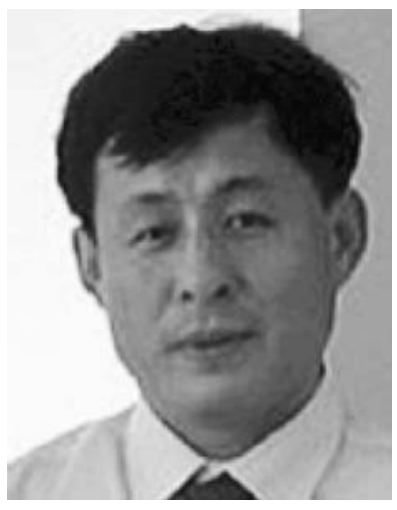

Zhihua Wang received the B.S., M.S., and Ph.D. degrees from the Department of Electronic Engineering, Tsinghua University, Beijing, China, in 1983, 1985, and 1990, respectively. He became a Professor with the Department of Electronic Engineering and Vice Director of the Institute of Microelectronics of Tsinghua University in 1997 and 2000, respectively. He has served as an official member of the Commission $\mathrm{C}$ of China National Commission of URSI starting in 1998 and the Chairman of the IEEE Solid-State Circuits Society Beijing Chapter. His major research fields are the design methodology and design automation of integrated circuits and systems, design of integrated circuits for communication, and high-speed real-time signal processing.

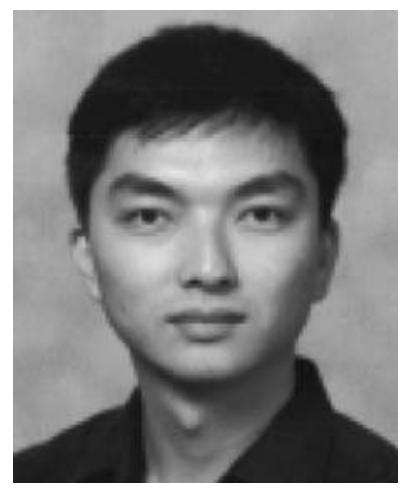

Chunsheng Liu (S'00-M'03) received the B.S. and M.S. degrees in electronic engineering from Tsinghua University, Beijing, China, in 1997 and 2000, respectively, and the $\mathrm{Ph} . \mathrm{D}$. degree in electrical and computer engineering from Duke University, Durham, NC, in 2003. He is currently an Assistant Professor of computer and electronics engineering with the University of NebraskaLincoln, Lincoln, NE. His research interests include verylarge-scale-integration design, testing, and fault diagnosis. He is currently working in the areas of NoC design and testing. Dr. Liu is a member of Association for Computing Machinery (ACM) and the ACM Special Interest Group on Design Automation. 